\title{
The new design of the THz streak camera at PSI
}

\author{
I. Gorgisyan,,${ }^{a, b, *}$ P.N. Juranić, ${ }^{a}$ R. Ischebeck, ${ }^{a}$ A. Stepanov, ${ }^{a}$ V. Schlott, ${ }^{a}$ C. Pradervand, ${ }^{a}$ L. Patthey, ${ }^{a}$ \\ M. Radović, ${ }^{a}$ R. Abela, ${ }^{a}$ C. P. Hauri, ${ }^{\mathrm{a}, \mathrm{b}}$ B. Monoszlai, ${ }^{\mathrm{a}, \mathrm{c}}$ R. Ivanov, ${ }^{\mathrm{d}}$ P. Peier, ${ }^{\mathrm{d}}$ J. Liu, ${ }^{\mathrm{e}}$ T. Togashi, ${ }^{\mathrm{f}}$ S. \\ Owada, ${ }^{g}$ K. Ogawa, ${ }^{g}$ T. Katayama, ${ }^{\mathrm{g}}$ M. Yabashi, ${ }^{\mathrm{g}}$ and L. Rivkin ${ }^{\mathrm{a}, \mathrm{b}}$

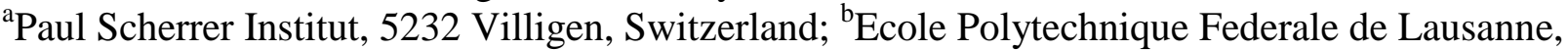 \\ 1015 Lausanne, Switzerland; ' ${ }^{\mathrm{C}}$ University of Pécs, 7624 Hungary; ${ }^{\mathrm{d}}$ Deutsches Elektronen-

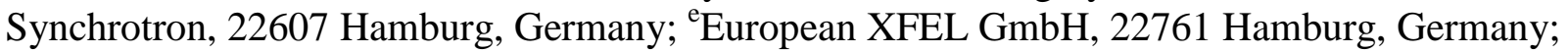 \\ ${ }^{\mathrm{f} J a p a n}$ Synchrotron Radiation Research Institute, Sayo, Hyogo 6795198, Japan; ${ }^{\mathrm{g}}$ RIKEN Spring-8 \\ Center, Sayo, Hyogo 6795148, Japan
}

\begin{abstract}
SwissFEL is the Free Electron Laser (FEL) facility under construction at the Paul Scherrer institute (PSI), aiming to provide users with X-ray pulses of lengths down to 2 femtoseconds at standard operation. The measurement of the length of the FEL pulses and their arrival time relative to the experimental laser is crucial for the pump-probe experiments carried out in such facilities. This work presents a new device that measures hard X-ray FEL pulses based on the THz streak camera concept. It describes the prototype setup called pulse arrival and length monitor (PALM) developed at PSI and tested in Spring-8 Angstrom Compact Free Electron Laser (SACLA) in Japan. Based on the first results obtained from the measurements, we introduce the new improved design of the second generation PALM setup that is currently under construction and will be used in SwissFEL photon diagnostics.

Keywords: THz Streak Camera, Free Electron Lasers, Atomic clusters, Instrumentation, Measurement, Time-of-Flight Spectrometer, Hard X-Rays, Photon diagnostics
\end{abstract}

\section{INTRODUCTION}

Free Electron Lasers provide extremely short photon pulses in both soft and hard X-ray regions with intensities of orders of magnitude higher than the 3rd generation synchrotron light sources. Typical experiments performed at FEL facilities around the world $[1,2,3]$ are pump-probe experiments where the sample is pumped by the experimental laser and then probed by the FEL radiation. These experiments use short-pulsed coherent X-ray radiation to achieve high-resolution measurements. For the FEL facilities where the radiation is created by self-amplified spontaneous emission (SASE) the temporal properties of the photon pulses vary largely from pulse to pulse. Particularly, the jitter in pulse length and arrival time with respect to the pump laser affects the resolution of pump-probe experiments. This stimulates the advancement of photon diagnostics in such facilities to provide comprehensive and accurate measurements of photon pulse lengths and arrival times. Providing accurate photon diagnostics at femtosecond scales, however, is quite challenging.

Many methods were proposed and developed during past few decades for both arrival time and pulse length measurements. Various techniques are utilized for measuring the relative arrival time of photon pulses, such as transmission/reflectivity spectral and spatial encoding [4, 5, 6, 7], THz streak camera for soft X-rays [8, 9] and others $[10,11]$. These methods can provide measurements with highest resolution of sub-10 femtoseconds. Different techniques were recently developed for photon pulse length measurements as well [11,12, 13, 14]. A theory using the THz streak camera concept was proposed for simultaneous high resolution measurement of pulse length and arrival time [15]. With this technique a resolution of sub-10 femtoseconds was achieved at FLASH for soft X-rays [8,9]. Among the mentioned systems $\mathrm{THz}$ streak camera is potentially capable of simultaneously measuring the pulse length and the arrival time of FEL pulses with a few femtoseconds resolution. The applicability of this method for hard X-ray photon pulse length measurement has not been experimentally demonstrated so far.

SwissFEL at PSI that is currently under construction will provide hard X-ray photon pulses with energies tunable from 2 $\mathrm{keV}$ to $12.4 \mathrm{keV}$ [16]. The pulse arrival and length monitor (PALM) developed at PSI [17] addresses the challenge of measuring the length and relative arrival time of pulses delivered by SwissFEL with a few femtoseconds accuracy. The

*ishkhan.gorgisyan@psi.ch; phone +4156310 4589

Advances in X-ray Free-Electron Lasers Instrumentation III, edited by Sandra G. Biedron Proc. of SPIE Vol. 9512, 95120D · @ 2015 SPIE · CCC code: 0277-786X/15/\$18

doi: $10.1117 / 12.2182204$

Proc. of SPIE Vol. 9512 95120D-1 
prototype PALM setup that was used in SACLA [18] for hard X-ray photon pulse measurements provided encouraging results and pointed the ways to improve the setup [19]. These results are used to design the second generation PALM that can perform measurements with higher accuracy.

\section{CONCEPT}

In a conventional streak camera the light pulses hit a photocathode to generate electron bunches. These bunches are then accelerated and transversely deflected by a time-varying field. Electrons with different arrival times are deflected differently and are registered on the screen. From the transverse distribution on the screen the temporal properties of the photon pulse can be obtained. Due to a large energy dispersion of the electrons from the cathode the resolution of the conventional streak camera is only few hundreds of femtoseconds [20,21]. To eliminate this factor the THz streak camera utilizes a gas as a source of electrons which is ionized by an X-ray photon pulse. The ionization is localized in the external $\mathrm{THz}$ field which streaks the energy spectrum of the photoelectrons. Figure 1 illustrates the concept of $\mathrm{THz}$ streaking. Due to the time-varying vector potential the spectrum of the streaked electrons shown in green contains information about the temporal profile and the energy spectrum of the pulse shown in blue in the figure. The figure also demonstrates how the position of the spectrum in green will change in energy depending on when the pulse in blue arrives in time.

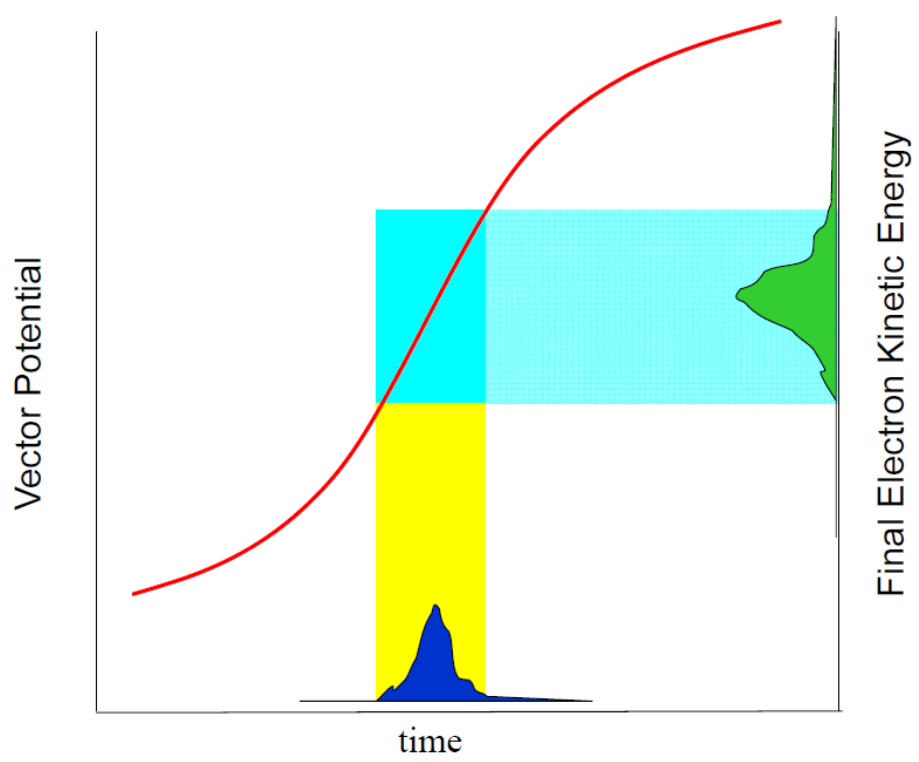

Figure 1. An illustration of the THz streaking concept. The blue peak in time scale represents the photon arrival (photoionization) time and the green peak is the final kinetic energy spectrum of electrons streaked by the $\mathrm{THz}$ vector potential (red curve).

The concept of the THz streak camera and the theory behind it is well explained in [15, 22, 23]. This work presents short summary of the theory of the THz streak camera mostly based on derivations in [8].

In these derivations we use semi-classical approach where we assume that the photoionization process is instantaneous. When the photoelectron is produced in absence of the external electric field its initial kinetic energy is $K_{i}=h v-W$, where the first term on the right hand side is the energy of the ionizing photon and the second term is the binding energy of the electron. Now we introduce an external $\mathrm{THz}$ field which is a linearly polarized electromagnetic wave with electric field strength given as $E_{T H z}(\mathrm{t})=E_{0} \cos \left(\omega_{T H z} \mathrm{t}+\phi\right)$. The electrons born in presence of the THz pulse will interact with the external field. The final kinetic energy of an electron propagating in such field will be

$$
K_{f}=K_{i}+2 U_{p} \sin ^{2}\left(\phi_{0}\right) \pm \sqrt{8 K_{i} U_{p}} \sin \left(\phi_{0}\right)
$$


Here $\phi_{0}$ is the phase of the electric field at the instant of ionization and $U_{p}$ is the ponderomotive potential written as

$$
U_{p}=\frac{e^{2} E_{0}^{2}}{4 m_{e} \omega_{T H z}^{2}}
$$

where $e$ and $m_{e}$ are the electron charge and mass respectively. When the electron is ionized by an X-ray photon its initial kinetic energy is of the order of hundreds of eVs up to several keVs while the ponderomotive potential is only several electron Volts so $U_{p} \ll K_{i}$. This means that the second term on the right hand side of (1) can be neglected with respect to the other terms. Therefore as it follows from expression (1) the final kinetic energy of the electron depends only on the phase of the vector potential at the moment the electron was ionized. If the electrons are ionized by photons of the same energy their final energy will vary depending on the time they were ionized. When the gas is ionized by a photon pulse with particular energy spectrum the created photoelectrons will initially have the same spectrum shifted by the constant of ionization potential. The central energy of the electron spectrum will be shifted in presence of the external field and this shift will depend on the moment when the ionization took place with respect to $\mathrm{THz}$ vector potential. By measuring the shift of the final kinetic energy spectrum of the electrons one can know when the ionization took place and therefore when the photon pulse arrived relative to the $\mathrm{THz}$ pulse. On the other hand as the electrons ionized by different parts of the photon pulse will be born at different times they will experience different vector potentials and therefore will have different final kinetic energies. The final energy spectrum of the photoelectrons born in the external time-varying field will be then the convolution of the photon pulse energy spectrum and the temporal profile. Based on this fact one can obtain information about the X-ray photon pulse length knowing the energy spectra of the electrons with and without the external streaking field. The calculations show that the rms width of the streaked electron spectrum can be written as

$$
\sigma_{\text {streak }}^{2}=\sigma_{0}^{2}+\tau_{X}^{2}\left(\mathrm{~s}^{2} \pm 4 \mathrm{cs}\right) .
$$

Here $\sigma_{0}$ is the rms width of the electron spectrum in absence of the THz field and $\tau_{X}$ is the rms length of the X-ray photon pulse. The terms $s$ and $c$ are respectively the THz streaking strength and the linear energy chirp along the photon pulse. Two opposite signs in (3) correspond to the electrons propagating along the vector potential and in the opposite direction. When the X-ray pulse is chirped in energy the spectra of the photoelectrons should be measured in two opposite directions corresponding to the signs in (3). Having the two streaked spectra and the non-streaked spectrum the photon pulse length can be calculated:

$$
\tau_{X}=\sqrt{\frac{\sigma_{\text {streak } 1}^{2}-\sigma_{0}^{2}+\sigma_{\text {streak } 2}^{2}-\sigma_{0}^{2}}{2 s^{2}}},
$$

where $\sigma_{\text {streak } 1}$ and $\sigma_{\text {streak } 2}$ are the streaked spectra of the electrons moving along the vector potential and opposite to it.

\section{MEASUREMENTS WITH THE PROTOTYPE SETUP}

The prototype PALM setup was designed and built at PSI to perform pulse length and arrival time measurements of hard $\mathrm{X}$-ray photon pulses based on THz streak camera concept. Figure 2 shows the outline of the setup. The THz beam enters the chamber through a $\mathrm{THz}$ window. A parabolic mirror is positioned inside the chamber to focus the $\mathrm{THz}$ beam on the interaction point. The mirror has a small aperture to let the X-Ray pulses through. The gas jet comes vertically down from a nozzle positioned above the interaction point. The gas jet, the $\mathrm{THz}$ beam and the $\mathrm{X}$-ray pulse need to be overlapped in time and space. After the overlap is achieved the gas jet is ionized by the X-ray pulse and the produced photoelectrons are then streaked by the vector potential of the THz pulse. The prototype PALM setup utilizes two ETF20 Kaesdorf electron time-of-flight (eTOF) spectrometers that measure the spectra of the photoelectrons. As indicates figure 2 the two eTOFs are positioned on both sides of the chamber in plane of the THz field polarization. They both point to the interaction region and due to the fact that they face to opposite directions they register electrons travelling parallel to the direction of the vector potential and opposite to it. 


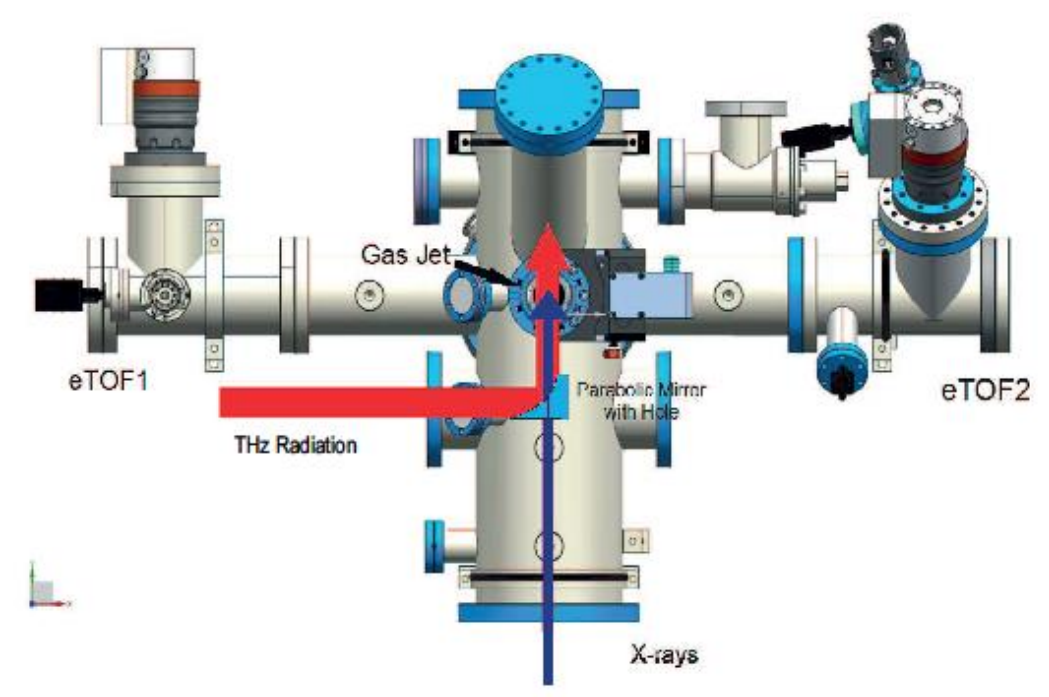

Figure 2. Schematic view of the prototype PALM setup. The red arrow represents the THz beam while the blue line corresponds to the X-ray beam.

The prototype setup was used to perform measurements at SACLA- hard X-ray FEL facility in Japan [18]. The photon pulse lengths and arrival times were measured for photon energies ranging from $5 \mathrm{keV}$ up to $12.4 \mathrm{keV}$ (1 Angstrom). The THz streaking measurements performed at SACLA at such high photon energies are the first of its kind.

The THz beam used in PALM was generated by a $\mathrm{LiNbO}_{4}$ crystal. The generation setup used the experimental laser at SACLA with $800 \mathrm{~nm}$ wavelength and $7 \mathrm{~mJ}$ pulse energy. The generated pulse frequency was $0.52 \mathrm{THz}$ with electric field strength of about $5 \mathrm{MV} / \mathrm{m}$. Xenon gas that was used in the setup has the highest photoionization cross-section among the noble gases at high photon energies due to atomic clusters formation in the pulses of the gas [24]. The Xe clusters were injected into the interaction region by a LaserLab Amsterdam piezo cantilever valve [25] synchronized to the $30 \mathrm{~Hz}$ repetition rate at SACLA.

During the measurements we used photon energies from $5 \mathrm{keV}$ to $10 \mathrm{keV}$ with $1 \mathrm{keV}$ steps and an energy of $12.4 \mathrm{keV}$. For the photon energies from $5 \mathrm{keV}$ to $10 \mathrm{keV}$ we also used a monochromator, which improves the bandwidth of the photon pulses from $0.3 \%-0.5 \%$ to about $0.01 \%$ at the cost of lowering the pulse energy by a factor of about 50. But even with reduced number of photons we still had enough signal due to Xe clusters formed inside the gas jet. For each photon energy value the measurements had the following order: 1) The photoelectrons were retarded to different final kinetic energies by changing the drift tube voltage of the eTOFs to obtain a time-to-energy calibration of the eTOFs for the particular photon energy. 2) The next step was a delay scan of the THz pulse with respect to the FEL pulse to map out the vector potential and electric field of the $\mathrm{THz}$ pulse. Data analysis of this scan provided information about the streaking strength or the linear slope (term $s$ introduced in equation 3) and the highest sensitivity area (dynamic range) along the vector potential which was about $600 \mathrm{fs}$. 3) After obtaining the range of the linear slope 10000 spectra were measured at the middle point of the slope assuring biggest possible dynamic range. The spread of the photon pulses along the linear slope of the vector potential defines directly the arrival time jitter of the FEL pulses. Another 10000 spectra were taken without the $\mathrm{THz}$ beam to be able to compare the streaked and non-streaked spectra for the photon pulse length estimations. The results obtained from these measurements indicate that the arrival time of the photon pulses was measured with an estimated accuracy in range of $4 \mathrm{fs}-9 \mathrm{fs}$ varying with the photon energy [19]. As the photon pulse lengths were calculated by comparing the averages of the streaked and unstreaked spectra the spectral width jitter of the photon pulses induced an additional error in the calculations. The first results revealed the strong and weak parts of the current setup and showed the way to further improve the measurement technique for the next generation PALM. 


\section{NEXT GENERATION SETUP}

Based on the results from the measurements at SACLA the next generation PALM setup is currently being developed at PSI with a new design that should improve its performance and achieve better accuracy for pulse length and arrival time measurements. Figure 3 shows the schematic view of the new design. A parabolic mirror which is inserted into the chamber focuses the $\mathrm{THz}$ beam on the interaction point. The main improvement of the next setup with respect to the prototype PALM is that the new design has two interaction points. Two piezo valves inject the gas jets into the chamber in two different positions indicated by the red arrows in figure 3.

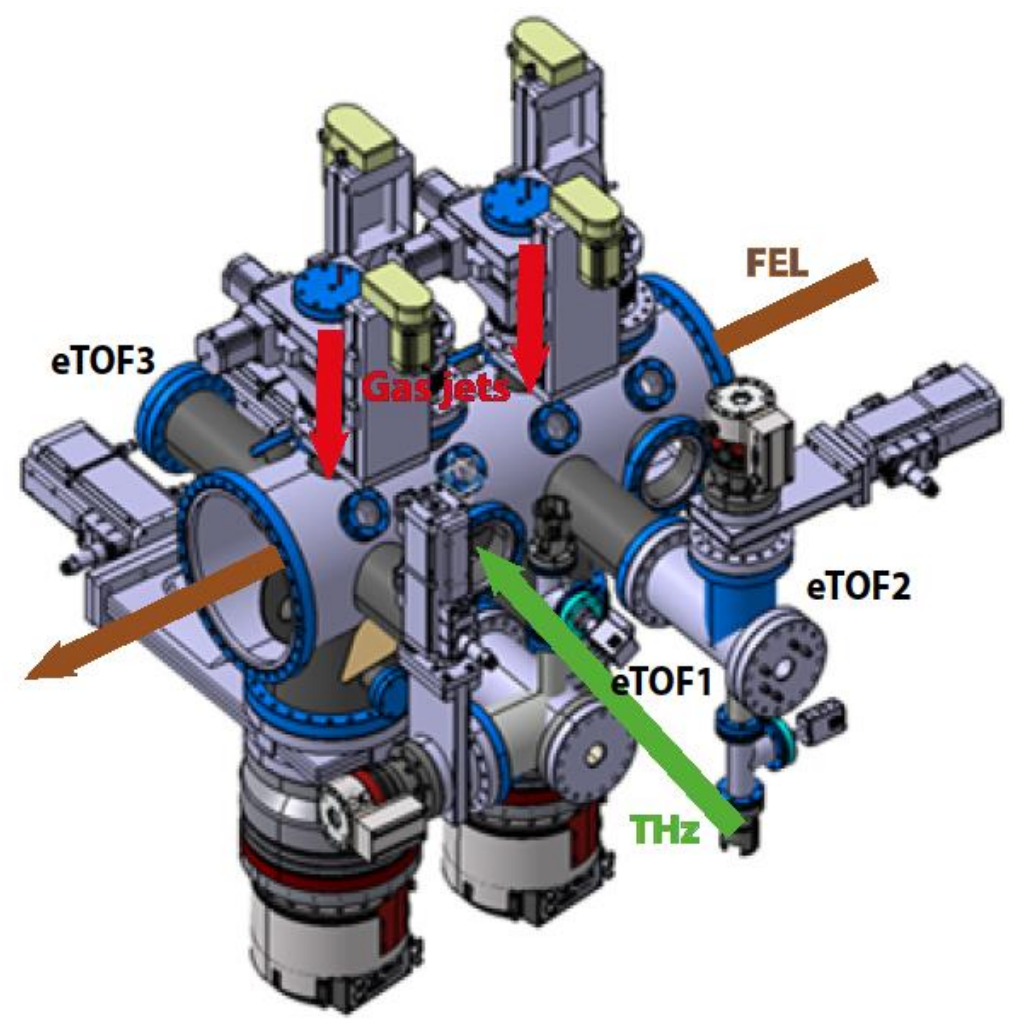

Figure 3. Outline of next generation PALM setup with two interaction regions. The brown and green arrows show the FEL and $\mathrm{THz}$ beams respectively, the two gas jets are shown by the red arrows.

Having the gas jets synchronized with the FEL and THz pulses we observe photoionization at two different points. At the first interaction region (upstream) the FEL ionizes the gas without the THz pulse which means that the produced photoelectrons are not streaked and their energy spectrum mimics the spectrum of the photon pulse. At the second interaction region the $\mathrm{THz}$ pulse is present and the spectrum of the photoelectrons is streaked and is registered by the two eTOFs from opposite sides. This design allows the simultaneous registration of the streaked and non-streaked spectra of the electrons produced by the same FEL pulse. This makes the measurements insensitive to the spectral width jitter of the photon pulses from shot to shot which is the main source of the error in the analysis. Eliminating this error will substantially increase the pulse length measurement accuracy compared to the prototype design which measures the pulse length by comparison of the streaked and unstreaked spectra averaged over many shots.

The presence of two interaction points means also that the setup will be an online monitor measuring the FEL pulse lengths on single-shot basis. The measurements of the PALM setup are non-invasive as the gas jets are almost transparent for the FEL pulses. Propagation through the chamber does not change the characteristics of the FEL pulses and they can be used for the experiments of the users.

The next generation PALM has the possibility of THz streaking at both interaction zones. The flexible design allows two $\mathrm{THz}$ beams simultaneously entering the chamber and being focused on the two interaction points. This is particularly 
useful for the cases when the FEL arrival time relative to the experimental laser (THz beam) varies largely and not all the pulses arrive at the linear area of the vector potential where the pulse length measurements are the most sensitive. Having two THz beams inside the chamber will double the dynamic range of the detector. The idea is to shift the phase of the two THz pulses by $90^{\circ}$ with respect to each other. Figure 4 illustrates the concept of this method. If the photon pulse arrives at the maximum of one vector potential it will still be at the linear part of the other one which means that at least at one of the interaction regions the electron spectrum will be well streaked and a measurement will be performed with the highest sensitivity. To define which interaction point had higher sensitivity measurement a rougher timing tool is required which should be synchronized with the PALM measurements. It is also worth noting that to have single shot measurements in this case the setup would require also the spectrum of the photon pulse or the photoelectrons without streaking. This can be achieved either by measuring the photon spectrum by a spectrometer or by adding another interaction region without $\mathrm{THz}$ in PALM setup.

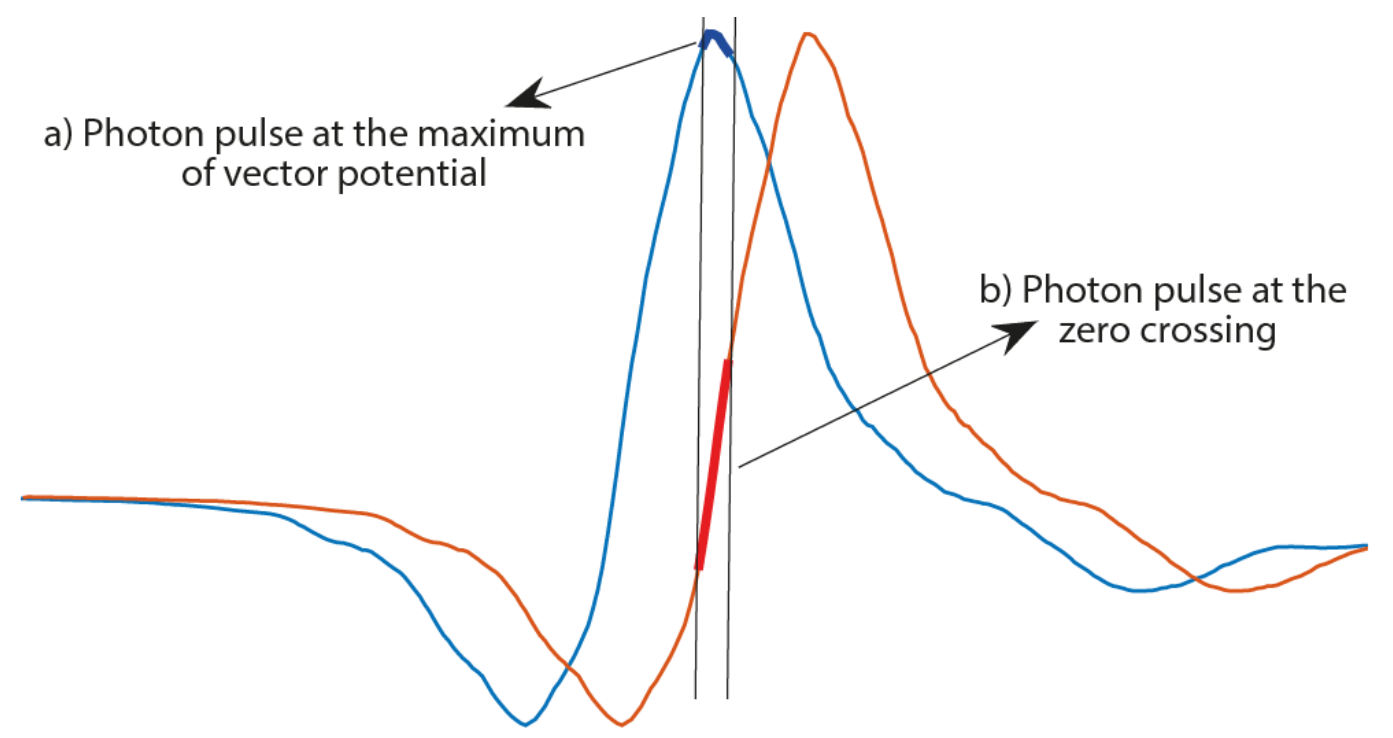

Figure 4. The concept of PALM with large dynamic range. The blue and red curves show the vector potentials that are offset by $90^{\circ}$ and correspond to the shape of the THz pulses used at prototype PALM. Even when the photon pulse is at the maximum of one vector potential (a) it is on the linear part of the second one (b).

The new design can also utilize two different $\mathrm{THz}$ pulses with different frequencies and streaking strengths. In this case the low-frequency pulse can roughly define the arrival time of the photon pulse and compare it with the arrival of the high-frequency pulse. If the FEL pulse arrives at the linear part of the high-frequency $\mathrm{THz}$ pulse it will be possible to measure the pulse length and the arrival time with higher accuracy. This measurement mode is not single-shot but can provide accurate measurements due to the high-frequency $\mathrm{THz}$ pulse with high field strength.

The new design of the next generation PALM can perform single-shot pulse length measurements with better accuracy as it excludes the contribution of the spectral width jitter of the photon pulses. The flexibility of the new design allows measurements in different modes depending on the task. It can measure with larger dynamic range in case of a big arrival time jitter or it can be pushed to the limits by using stronger THz fields at high frequencies. Overall, the updated PALM design will allow more flexibility in measurements and will measure the photon pulses with improved accuracy.

\section{CONCLUSION}

The PSI-developed PALM device will measure the arrival time and pulse length of hard X-rays at SwissFEL. The prototype setup has already proved the concept of measuring the pulse length and arrival time of hard X-ray FEL pulses using $\mathrm{THz}$ streaking technique. The next generation PALM has an improved design and will be able to perform measurements with better accuracy. It will be used in SwissFEL photon diagnostics as an online non-invasive timing tool. 


\section{REFERENCES}

[1] Ackermann, W. et. al., "Operation of a free-electron laser from the extreme ultraviolet to the water window," Nature Photonics, vol. 1, p. 336, (2007).

[2] Emma, P. et. al., "First lasing and operation of an angstrom-wavelength free-electron laser," Nature Photon, vol. 4, p. 641, (2010).

[3] Pile, D. et. al., "X-rays first light from SACLA," Nature Photon, vol. 5, p. 436, (2011).

[4] Bionta, M. et. al., "Spectral encoding of x-ray/optical relative delay," Optics Express, vol. 19, p. 21855, (2011).

[5] Krupin, O. et. al., "Temporal cross-correlation of x-ray free electron and optical lasers using soft $\mathrm{x}$-ray pulse induced transient reflectivity," Optics Express, vol. 20, p. 11396, (2012).

[6] Harmand, M. et. al., "Achieving few-femtosecond time-sorting at hard X-ray free-electron lasers," Nature Photon., vol. 7, pp. 215-218, (2013).

[7] Hartmann, N., Helml, W., Galler, A., Bionta, M. R., Grünert, J., Molodtsov, L. S., Ferguson, K. R., Schorb, S., Swiggers, M. L., Carron, S., Bostedt, C., Castagna, J. C., Bozek, J., Glownia, J. M., Kane, D. J., Fry, A. R., White, W. E., Hauri, C. P., Feurer, T. and Coffee, R. N., "Sub-femtosecond precision measurement of relative X-ray arrival time for free-electron lasers," Nat Photon, 8:706-709, (2014).

[8] Frühling, U. et. al., "Single-Shot Terahertz-Field-Driven X-ray Streak Camera," Nature Photon, vol. 3, p. 353, (2009).

[9] Grguras, I. et. al., "Utrafast X-ray pulse characerization at free electron lasers," Nature Photon, vol. 6, p. 852, (2012).

[10] Tavella, F. et. al., "Few-femtosecond timing at fourth-generation X-ray light sources," Nature Photon, vol. 5, p. 162, (2011).

[11]Duesterer, S. et. al., "Femtosecond x-ray pulse length characterization at the Linac Coherent Light Source freeelectron laser," New J. PHys, vol. 13, p. 093024, (2011).

[12] Drescher, M. et. al., "X-ray pulses approaching the attosecond frontier," Science, vol. 291, p. 1923, (2001).

[13] Riedel, R. et. al., "Single-shot pulse duration monitor for exterme ultraviolet and X-ray free electron lasers," Nature Comm., vol. 4, p. 1731, (2013).

[14] Helml, W. et al., "Measuring the temporal structure of few-femtosecond free-electron laser X-ray pulses directly in the time domain", Nature Photon., vol. 8 (12) pp. 950-957, (2014).

[15] Itatani, J., Quéré, F., Yudin, G. L., Ivanov, M. Yu., Krausz, F. and Corkum, P. B., "Attosecond streak camera," Phys. Rev. Lett., 88:173903, (2002).

[16] Oberta, P. et. al., "The SwissFEL facility and its preliminary optics beamline layout," Proceedings of SPIE, vol. 8079, p. 807805, (2011)

[17] Juranic, P. et. al., "A scheme for a shot-to-shot, femtosecond-resolved pulse length and arrival time measurement of free electron laser x-ray pulses that overcomes the time jitter problem between the FEL and the laser," JINST, vol. 9, p. P03006, (2014).

[18]Tono, K., Togashi, T., Inubushi, Y., Sato, T., Katayama, T., Ogawa, K., Ohashi, H., Kimura, H., Takahashi, S., Takeshita, K., Tomizawa, H., Goto, S., Ishikawa, T. and Yabashi M., "Beamline, experimental stations and photon beam diagnostics for the hard x-ray free electron laser of SACLA," New Journal of Physics, vol. 15, p. 083035, (2013).

[19] Juranic, P. N., Stepanov, A., Ischebeck, R., Schlott, V., Pradervand, C., Patthey, L., Radovic, M., Gorgisyan, I., Rivkin, L., Hauri, C. P., Monoszlai, B., Ivanov, R., Peier, P., Liu, J., Togashi, T., Owada, S., Ogawa, K., Katayama, T., Yabashi, M. and Abela R., "High-precision x-ray fel pulse arrival time measurements at SACLA by a THz streak camera with Xe clusters," Opt. Express, 22(24):30004-30012, (2014).

[20] Shakya, M.M. and Chang, Z., "Achieving 280 fs resolution with streak camera by reducing deflection dispersion," Appl. Phys. Lett. 87, (2005).

[21]Feng, J., Shin, H. J., Nasiatka, J. R., Wan, W., Young, A. T., Huang, G., Comin, A., Byrd, J. and Padmore, H. A., "An x-ray streak camera with high spatio-temporal resolution," Applied Physics Letters, 91(13), (2007).

[22] Uiberecker M. et. al., "Attosecond Metrology with Controlled Light Waveforms," Laser Physics, vol. 15, p. 195, (2005).

[23] Quéré, F., Mairesse, Y. and Itatani, J., “Temporal characterization of attosecond XUV fields,” Journal of Modern Optics, 52(2-3):339-360, (2005).

[24] Hagena, O. F. and Obert, W., "Cluster formation in expanding supersonic jets: Effect of pressure, temperature, nozzle size, and test gas," The Journal of Chemical Physics, 56(5), (1972). 
[25] Irimia, D., Dobrikov, D., Kortekaas, R., Voet, H., van den Ende, D. A., Groen, W. A. and Janssen, M. H. M., “A short pulse (7 micros FWHM) and high repetition rate $(\mathrm{dc}-5 \mathrm{kHz})$ cantilever piezovalve for pulsed atomic and molecular beams," Rev. Sci. Instrum. 80(11), 113303 (2009). 